\title{
The Effects of Sildenafil on Fetal Doppler Indices: A Systematic Review and Meta-Analysis
}

\author{
Maryam Damghanian 1(D), Farnaz Farnam ${ }^{2}$ iD, Roghieh Kharaghani ${ }^{3 *(D)}$ \\ 1. Nursing and Midwifery Care Research Center, School of Nursing and Midwifery, Tehran University of Medical \\ Sciences, Tehran, Iran \\ 2. Dept. of Reproductive Health, School of Nursing and Midwifery, Tehran University of Medical Sciences, Tehran, Iran \\ 3. Dept. of Midwifery, School of Nursing and Midwifery. Zanjan University of Medical Sciences, Zanjan, Iran
}

\begin{tabular}{c}
\hline Article Info \\
\hline dof 10.30699/jambs.28.131.296 \\
\hline Received: $2020 / 04 / 25 ;$ \\
Accepted: $2020 / 08 / 12 ;$ \\
Published Online: 20 Oct 2020 \\
\hline Use your device to scan and read the \\
article online \\
a \\
(a)
\end{tabular}

Corresponding Information: Roghieh Kharaghani,

Dept.of Internal Medicine, Vali-e-Asr Hospital, School of Medicine, Zanjan University of Medical Sciences, Zanjan, Iran

E-Mail:

R.kharaghani@zums.ac.ir

\begin{abstract}
Background \& Objective: Sildenafil citrate is a potential new strategy for the management of intrauterine growth restriction (IUGR) and preeclampsia, although its efficacy still needs to be approved. Accordingly, the aim of this study was to systematically assess the effectiveness of sildenafil on improving fetal Doppler indices, as well as the most common adverse effects of sildenafil.
\end{abstract}

Materials \& Methods: Online databases, i.e., PubMed, Web of Science, Scopus, Embase, and Cochrane Reviews, were searched from their date of creation up to 26 Jan 2019. We conducted two meta-analyses, one for fetal Doppler indices and the other for adverse events of sildenafil. Eligible studies were randomized controlled trials (RCTs), in which the effects of sildenafil on fetal Doppler indices have been evaluated. The quality of studies was appraised through the five components of the Cochrane checklist (for quality appraisal of RCT studies) by two of the authors.

Results: Out of 1,087 reviewed sources, seven studies were included for metaanalysis. The following results were observed for the effect of sildenafil on the umbilical artery (UA): A significant effect on the umbilical artery pulsatility index (UA PI) $(P=0.03)$ was observed in dosages more than $60 \mathrm{mg} / \mathrm{d}(P=0.008)$. Sildenafil was effective in UA PI of the individuals suffering from eclampsia $(P=0.008)$; however, no significant effect was observed on IUGR disorder. Furthermore, sildenafil had no effect on the umbilical artery systolic/diastolic (UA S/D) ratio.Sildenafil was not effective on the middle cerebral artery pulsatility index (MCA PI) or on the middle cerebral artery systolic/diastolic (MCA S/D) ratio. Regarding adverse events, headaches occurred significantly more in mothers consuming sildenafil $(P=0.03)$.

Conclusion: Obviously, we need more accurate RCTs in this issue before any decision can be made.

Keywords: Sildenafil, Doppler indices, Intrauterine growth restriction, Preeclampsia, Meta-analysis

\section{Introduction}

In the first half of pregnancy, uteroplacental arteries (especially spiral arteries) undergo a series of pregnancy-specific changes to meet the requirements of the fetus. Spiral artery modification reduces maternal blood flow resistance and increases uteroplacental perfusion (1). Reduced trophoblast invasion and the absence of pregnancy-specific changes of uteroplacental arteries have a crucial role in intrauterine growth restriction (IUGR) and are often combined with preeclampsia. Also, in a normal pregnancy, the trophoblast produces nitric oxide (NO), which acts as a vasodilator in fetoplacental circulation. It has been shown that NO can improve perfusion (in IUGR pregnancies) and preeclampsia by relaxing the arteries and improving oxygen and nutritional supplies (2). Consequently, it is assumed that sildenafil citrate can be a potential strategy to aid women suffering from IUGR and preeclampsia during pregnancy by releasing $\mathrm{NO}(3)$.

Sildenafil citrate in tablet form and under the name Viagra was initially used to treat erectile dysfunction in men. The physiologic mechanism of sildenafil involves the release of NO, and it enhances the effect of NO by inhibiting phosphodiesterase type 5 (PDE5). This process can relax the muscles of blood vessels and other areas of the body, such as the uterine vessel $(3,4)$. This finding has inspired many researchers to use sildenafil for the management of preeclampsia and IUGR (5-12). 
Due to an increase in the prevalence of preeclampsia, as well as the massive physical, psychological, and economic burdens of preeclampsia and IUGR, sildenafil can be of great interest as an innovative approach, even though its efficacy still needs to be approved (13-16). Every medication should be prescribed with extreme caution. To date, the use of sildenafil in human pregnancy has not been clinically approved; it is confined to research studies.

Unfortunately, due to the lack of adequate randomized controlled trials (RCTs), different reported outcomes, or unqualified research works, only two systematic reviews exist in this field. The first one evaluated the effect of sildenafil on IUGR with in vitro studies, and the other reported the adverse effects of sildenafil usage in pregnancy, along with some obstetrical and perinatal outcomes $(17,18)$. For evaluating sildenafil efficacy in pregnancy, one of the most reported outcomes has been Doppler ultrasonography indices. Two common Doppler indices for predicting and evaluating IUGR and preeclampsia are related to the umbilical artery (UA) and the middle cerebral artery (MCA) $(19,20)$.

In our search, the outcome that led to the recruitment of the most qualified RCTs was associated with Doppler ultrasonography indices. It seems that if the effect of sildenafil on fetal Doppler indices is known, then the effect of sildenafil on IUGR and preeclampsia can be predicted through evidence-based studies. It may also be useful for understanding the mechanism of effects more accurately.

Considering the importance of evidence-based research studies for clinical decision-making, this meta-analysis aimed to evaluate sildenafil's efficacy in improving fetal Doppler indices. It also describes the most common adverse effects of sildenafil. Specifically, the umbilical artery pulsatility index (UA $\mathrm{PI})$, umbilical artery systolic/diastolic (UA S/D) ratio, middle cerebral artery pulsatility index (MCA PI), and middle cerebral artery systolic/diastolic (MCA S/D) ratio were evaluated in mothers suffering from preeclampsia and IUGR during pregnancy.

\section{Materials and Methods}

\section{Search Strategy}

Online databases, i.e., PubMed, Web of Science, Scopus, Embase, and Cochrane Reviews, were searched from their date of creation up to 26 Jan 2019. The MeSH terms for "Sildenafil," "Pregnancy," "Preeclampsia," "IUGR," "Amniotic fluid," "Apgar score," and "mode of delivery" were extracted from PubMed.

\section{Selection Process}

Studies lacking control groups or that were not related to the main subject were excluded. Two of the authors reviewed the title and abstract of each paper individually and extracted the full text of the related studies and, ultimately, the required data. Disagreements about inclusion or exclusion were discussed and resolved by consensus or arbitration. The percent agreement of the two researchers was $97.5 \%$, and the Kappa statistic was $91.9 \%$. The extracted data included authors, year of publication, country, type of study, study population, age of mother, term of pregnancy upon receiving sildenafil, dose of received sildenafil, and population size in intervention groups and control groups, as well as indices related to fetal Doppler, UA, and MCA.

\section{Sub-Group Analysis}

Sub-groups of fetal Doppler indices (UA and MCA) were analyzed based upon the dose of the used medication, term of effect assessment, study population, and quality of the conducted studies.

\section{Adverse Events}

The reported adverse events included headache, hot flash, nausea and vomiting, epigastric pain, neurologic disorders, visual disturbances, dizziness, abruption, postpartum hemorrhage, eclampsia, and backache, as well as hemolysis, elevated liver enzymes, and low platelets (HELLP) syndrome. These were compared in both groups.

\section{Quality Appraisal of Studies}

The quality of each study was appraised based on the five components of the Cochrane checklist for quality appraisal of RCT studies, including random sequence generation, allocation concealment, blinding, incomplete reporting, and selective outcome reporting. According to the checklist, the studies were scored between 0 and 2 (high-risk, unknown, and low-risk). In cases where all the above-mentioned criteria were present, the respective study was selected as a highquality study. A score of 8 to 9 represented a mediumquality study; if a study did not meet more than two of the criteria (yielding a score of less than 8), it was categorized as a low-quality study. None of the studies were excluded due to their quality.

\section{Data Analyses}

RevMan 5.3 (Computer program, Version 5.3. Copenhagen: The Nordic Cochrane Centre, The Cochrane collaboration, 2015) was employed for the meta-analysis of data. The mean and SD of fetal Doppler indices, including fetal Doppler indices PI and the S/D ratios of UA and MCA, were extracted from the studies. Also, the adverse effects of sildenafil were extracted from qualified studies. Two meta-analyses were conducted on fetal Doppler indices and maternal adverse effects. The first meta-analysis on Doppler indices was conducted using standardized mean difference (SMD) and random effects. SMDs were measured per Cohen regulations: Any value lower or equal to 0.2 was denoted as a "minor effect"; a value between 0.2 and 0.8 was denoted as a "medium effect," and any value equal to or higher than 0.8 was denoted as a "major effect" (21). The $I^{2}$ statistic was employed 
for examination of the study's heterogeneity (22). In the second meta-analysis, the effect of sildenafil on maternal adverse events was analyzed using an odds ratio and the random effect between the two intervention and control groups. Also, Begg and Egger's statistical tests were used to measure publication bias $(23,24)$. The Ethical Committee approval and participants' informed consent were not required.

\section{Results}

\section{Selection Process}

A search among electronic resources yielded 1,084 references from databases and three from references of papers. Out of 1,087 reviewed sources, 804 studies were excluded for repetition, and 511 were excluded for other reasons. Moreover, 463 studies were not related to the main subject, and 48 were excluded from the systematic review and meta-analysis for not being a clinical trial. Out of 101 studies remaining for fulltext evaluation, 94 studies were excluded for not containing adequate data, and another two were excluded for not having any control or waiting groups. Ultimately, seven studies were included in the metaanalysis (Figure 1).

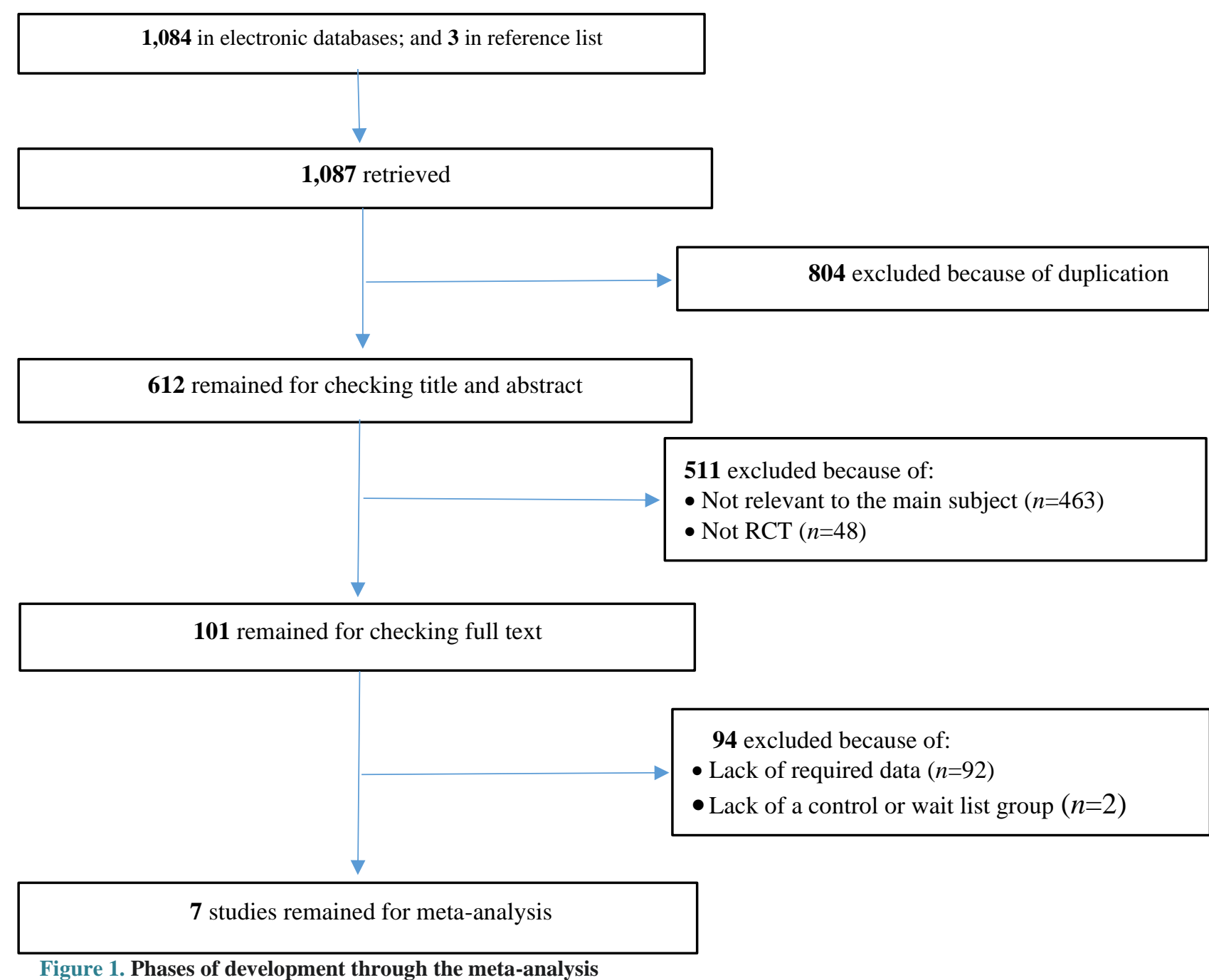

Figure 1. Phases of development through the meta-analysis

\section{Study Characteristics}

Seven papers met the required criteria for inclusion in this meta-analysis (3-9). The total number of participants in these studies was 361. Of these, 180 individuals were in intervention groups, and 181 individuals were in control groups. The mean ages of mothers in intervention and control groups were 26.4 and 28.6 years, respectively. The mean ages of pregnancy upon admission to the study in the intervention and control groups were 29.8 and 30.4 weeks, respectively. Most studies were conducted on IUGR (five studies), and the rest examined preeclampsia (two studies). The quality of most of the studies was high $(57 \%)$ (Table 1).

The funnel plots in both meta-analyses were symmetrical, and it seems that the probability of publication bias is very low. 
Table 1. Characteristics of included studies in the meta-analysis

\begin{tabular}{|c|c|c|c|c|c|c|c|c|c|c|}
\hline \multicolumn{3}{|c|}{ Author } & \multicolumn{2}{|c|}{ Year } & country & \multicolumn{2}{|c|}{ Design } & \multicolumn{3}{|c|}{ Study population } \\
\hline \multicolumn{3}{|c|}{ Vahid Dastjerdi } & \multicolumn{2}{|c|}{2012} & Iran & \multicolumn{2}{|c|}{ RCT } & \multicolumn{3}{|c|}{ IUGR } \\
\hline \multicolumn{3}{|c|}{ El-Sayed } & \multicolumn{2}{|c|}{2017} & Egypt & \multicolumn{2}{|c|}{ RCT } & \multicolumn{3}{|c|}{ IUGR } \\
\hline \multicolumn{3}{|c|}{ Samangaya } & \multicolumn{2}{|c|}{2009} & UK & \multicolumn{2}{|c|}{ RCT } & \multicolumn{3}{|c|}{ Preeclampsia } \\
\hline \multicolumn{3}{|c|}{ Shehata } & \multicolumn{2}{|c|}{2018} & Egypt & \multicolumn{2}{|c|}{ RCT } & \multicolumn{3}{|c|}{ IUGR } \\
\hline \multicolumn{3}{|c|}{ Trapani } & \multicolumn{2}{|c|}{2016} & Brazil & \multicolumn{2}{|c|}{ RCT } & \multicolumn{3}{|c|}{ IUGR } \\
\hline \multicolumn{3}{|c|}{ Trapani } & \multicolumn{2}{|c|}{2016} & Brazil & \multicolumn{2}{|c|}{$\mathrm{RCT}$} & \multicolumn{3}{|c|}{ Preeclampsia } \\
\hline Mag & & & 201 & & Egypt & rando & RCT & & IUGR & \\
\hline & Mate & al age & GA & veek) & & Sam & le size & & PI of Umbi & 1 Artery \\
\hline & Int. & Cont. & Int. & Cont. & & Int. & Cont. & & Int. & Cont. \\
\hline Vahid Dastjerdi & 25.6 & 32 & 35 & 35 & $50 \mathrm{mg}$ & 29 & 30 & Low & $1.01(0.13)$ & $\begin{array}{c}1.02 \\
(0.31)\end{array}$ \\
\hline El-Sayed & 26.3 & 28.1 & 29.7 & 29.3 & $50 \mathrm{mg}$ daily & 27 & 27 & High & $0.79(0.59)$ & $\begin{array}{l}0.14 \\
(0.5)\end{array}$ \\
\hline Samangaya & $28^{*}$ & $27 *$ & $31 *$ & $29 *$ & $20-80 \mathrm{mg}$ TDS & 17 & 18 & High & $1.17(0.22)$ & $\begin{array}{c}1.27 \\
(0.14)\end{array}$ \\
\hline Shehata & 30.4 & 30.7 & 29.5 & 30.1 & $20 \mathrm{mg}$ TDS & 23 & 23 & Moderate & $1.8(0.03)$ & $\begin{array}{c}1.9 \\
(0.03)\end{array}$ \\
\hline Trapani & 23.6 & 25.8 & 28.5 & 30.1 & $50 \mathrm{mg}$ single dose & 12 & 12 & High & $1.21(0.13)$ & $\begin{array}{c}1.40 \\
(0.11)\end{array}$ \\
\hline Trapani & 25.3 & 26.4 & 29.1 & 30.2 & $50 \mathrm{mg}$ TDS & 47 & 46 & High & $1.19(0.17)$ & $\begin{array}{c}1.38 \\
(0.13)\end{array}$ \\
\hline Maged & 27.3 & 28.7 & 27.4 & 28.1 & $20 \mathrm{mg}$ daily to $20 \mathrm{mg}$ TDS & 25 & 25 & Low & $1.88(0.02)$ & $\begin{array}{c}1.94 \\
(0.01)\end{array}$ \\
\hline
\end{tabular}

Abbreviations: RCT, randomized controlled trial; IUGR, intrauterine growth restriction; PI, pulsatility index; TDS, three times a day; GA, gestational age; SC, sildenafil citrate; Int, intervention; Cont, control; *Data are median.

\section{Subgroup Analyses}

\section{The Effects of Sildenafil on the Umbilical Artery Indices}

\section{Umbilical Artery Pulsatility Index}

The results yielded by the studies revealed that sildenafil significantly decreases UA PI to a value of 1.28 in the intervention group compared with the control group $(\mathrm{SMD}=-1.28 ; 95 \% \mathrm{CI},-2.44$ to -0.12 ; $P=0.03$ ) ( $\underline{\text { Table 2 }}$ ). The sildenafil effect on UA PI was observed in dosages of more than $60 \mathrm{mg} / \mathrm{d}$ $(\mathrm{SMD}=-.0 .94 ; 95 \% \mathrm{CI},-1.63$ to $-0.25 ; P=0.008)$, and in the durations of 24 hours $(\mathrm{SMD}=-1.24 ; 95 \%$ CI, -1.69 to $-0.80 ; P<0.001)$, two weeks (SMD= $0.90 ; 95 \% \mathrm{CI},-1.59$ to $-0.20 ; P=0.01$ ), and four weeks $(\mathrm{SMD}=-3.74 ; 95 \% \mathrm{CI},-4.68$ to -2.79 ; $P<0.001)$, but it was ineffective in the durations of two hours (Table 2 and Figure 2). From a study population perspective, sildenafil was effective in preeclampsia $(\mathrm{SMD}=-0.94 ; 95 \% \mathrm{CI},-1.63$ to -0.25 ; $P=0.008$ ), but had no effect on IUGR (Table 2 and Figure 3 ). From a study quality perspective, only medium quality studies showed such an effect
$(\mathrm{SMD}=-3.28 ; 95 \% \mathrm{CI},-4.18$ to $-2.37 ; P<0.001)$ (Table 2).

\section{Umbilical Artery Systolic/Diastolic Ratio}

Sildenafil exerted no effect on the UA S/D ratio. The effect was significant only in a four-week follow-up subgroup $(\mathrm{SMD}=-2.94 ; 95 \% \mathrm{CI},-3.76$ to $-2.12 ; P<0.001)$ and high quality studies $(\mathrm{SMD}=$ 1.47; 95\% CI, 0.86-2.07; $P<0.001$ ) (Table 2).

The Effect of Sildenafil on the Middle Cerebral Artery Indices

\section{Middle Cerebral Artery Pulsatility Index}

Sildenafil had no effect on MCA PI of the embryo. The effect was significant only in a two-hour followup $(\mathrm{SMD}=2.89 ; 95 \% \mathrm{CI}, 0.43-5.35 ; P=0.02)$, twoweek follow-up $(\mathrm{SMD}=57.83$; 95\% CI, 45.46-70.20; $P<0.001)$ and IUGR $(\mathrm{SMD}=2.89 ; 95 \% \mathrm{CI}, 0.43-$ $5.35 ; P=0.02)$ subgroups, as well as in low quality studies $\quad(\mathrm{SMD}=39.31 ; \quad 95 \% \quad \mathrm{CI}, \quad 30.89-47.74$; $P<0.001$ ) (Table 2). 
Table 2. The effects of sildenafil on the Doppler indices in different sub-groups of the study (based on SMD and random effect)

\begin{tabular}{|c|c|c|c|c|c|c|c|c|c|c|c|c|}
\hline \multirow{3}{*}{$\begin{array}{l}\text { Adverse } \\
\text { events }\end{array}$} & \multicolumn{6}{|c|}{ Umbilical Artery } & \multicolumn{6}{|c|}{ Middle Cerebral Artery } \\
\hline & \multicolumn{3}{|c|}{ PI } & \multicolumn{3}{|c|}{$\mathrm{S} / \mathrm{D}$ ratio } & \multicolumn{3}{|c|}{ PI } & \multicolumn{3}{|c|}{$\mathrm{S} / \mathrm{D}$ ratio } \\
\hline & SMD & $95 \% \mathrm{CI}$ & $\begin{array}{c}\mathrm{P}- \\
\text { value }\end{array}$ & SMD & $95 \% \mathrm{CI}$ & $\begin{array}{c}\text { P- } \\
\text { value }\end{array}$ & SMD & $95 \% \mathrm{CI}$ & $\begin{array}{c}\mathrm{P}- \\
\text { value }\end{array}$ & SMD & $95 \% \mathrm{CI}$ & $\begin{array}{c}\mathrm{P}- \\
\text { value }\end{array}$ \\
\hline \multicolumn{13}{|c|}{ Dose of the Sildenafil } \\
\hline $\begin{array}{c}50 \mathrm{mg} \\
\text { Single dose }\end{array}$ & -0.74 & $\begin{array}{c}-2.20 \\
0.71\end{array}$ & 0.32 & -0.14 & $\begin{array}{c}-0.78 \\
0.51\end{array}$ & 0.68 & -0.06 & $-0.57,0.44$ & 0.80 & 0.15 & $\begin{array}{c}-0.50 \\
0.80\end{array}$ & 0.65 \\
\hline $\begin{array}{c}20-60 \mathrm{mg} \\
\text { daily }\end{array}$ & -1.93 & $\begin{array}{c}-5.35 \\
1.49\end{array}$ & 0.27 & -0.73 & $\begin{array}{c}-5.04 \\
3.59\end{array}$ & 0.74 & 19.91 & $\begin{array}{c}-17.66 \\
57.47\end{array}$ & 0.30 & 1.06 & $\begin{array}{c}0.49 \\
1.63\end{array}$ & $<0.001$ \\
\hline $\begin{array}{c}>\text { than } 60 \mathrm{mg} \\
\text { daily }\end{array}$ & -0.94 & $\begin{array}{l}-1.63 \\
-0.25\end{array}$ & 0.008 & - & - & - & -0.20 & $-0.61,0.21$ & 0.34 & - & - & - \\
\hline \multicolumn{13}{|c|}{ Time of outcome assessment } \\
\hline 2 hours & -0.89 & $\begin{array}{c}-2.72 \\
0.94\end{array}$ & 0.34 & 0.67 & $\begin{array}{c}-0.90 \\
2.24\end{array}$ & 0.40 & 2.89 & $0.43,5.35$ & 0.02 & 0.62 & $\begin{array}{c}-0.27 \\
1.51\end{array}$ & 0.17 \\
\hline 24 hours & -1.24 & $\begin{array}{l}-1.69 \\
-0.80\end{array}$ & $<0.001$ & - & - & - & -0.20 & $-0.61,0.21$ & 0.34 & - & - & - \\
\hline 2 weeks & -0.90 & $\begin{array}{l}-1.59 \\
-0.20\end{array}$ & 0.01 & - & - & - & 57.83 & $45.46,70.20$ & $<0.001$ & - & - & - \\
\hline 4 weeks & -3.74 & $\begin{array}{l}-4.68 \\
-2.79\end{array}$ & $<0.001$ & -2.94 & $\begin{array}{c}-3.76,- \\
2.12\end{array}$ & $<0.001$ & - & - & - & - & - & - \\
\hline \multicolumn{13}{|c|}{ Study population } \\
\hline IUGR & -1.46 & $\begin{array}{c}-3.33 \\
0.41\end{array}$ & 0.13 & -0.52 & $\begin{array}{c}-2.89 \\
1.85\end{array}$ & 0.67 & 2.89 & $0.43,5.35$ & 0.02 & 0.62 & $\begin{array}{c}-0.27 \\
1.51\end{array}$ & 0.17 \\
\hline Preeclampsia & -0.94 & $\begin{array}{l}-1.63 \\
-0.25\end{array}$ & 0.008 & - & - & - & -0.20 & $-0.61,0.21$ & 0.34 & - & - & - \\
\hline \multicolumn{13}{|c|}{ Quality of the studies } \\
\hline High & -0.52 & $\begin{array}{c}-1.75 \\
0.72\end{array}$ & 0.41 & 1.47 & $\begin{array}{l}0.86 \\
2.07\end{array}$ & $<0.001$ & 0.18 & $-0.64,0.99$ & 0.67 & 1.06 & $\begin{array}{c}0.49 \\
1.63\end{array}$ & $<0.001$ \\
\hline Moderate & -3.28 & $\begin{array}{l}-4.18 \\
-2.37\end{array}$ & $<0.001$ & - & - & - & 39.31 & $30.89,47.74$ & $<0.001$ & - & - & - \\
\hline Low & -1.87 & $\begin{array}{c}-5.49 \\
1.75\end{array}$ & 0.31 & -1.53 & $\begin{array}{l}-4.27 \\
1.22\end{array}$ & 0.28 & 0.08 & $-0.57,0.72$ & 0.82 & 0.15 & $\begin{array}{c}-0.50 \\
0.80\end{array}$ & 0.65 \\
\hline Total effect & -1.28 & $\begin{array}{l}-2.44 \\
-0.12\end{array}$ & $\mathbf{0 . 0 3}$ & -0.52 & $\begin{array}{c}-2.89 \\
1.85\end{array}$ & 0.67 & 1.38 & $-0.22,2.98$ & 0.09 & 0.62 & $\begin{array}{c}-0.27 \\
1.51\end{array}$ & 0.17 \\
\hline
\end{tabular}

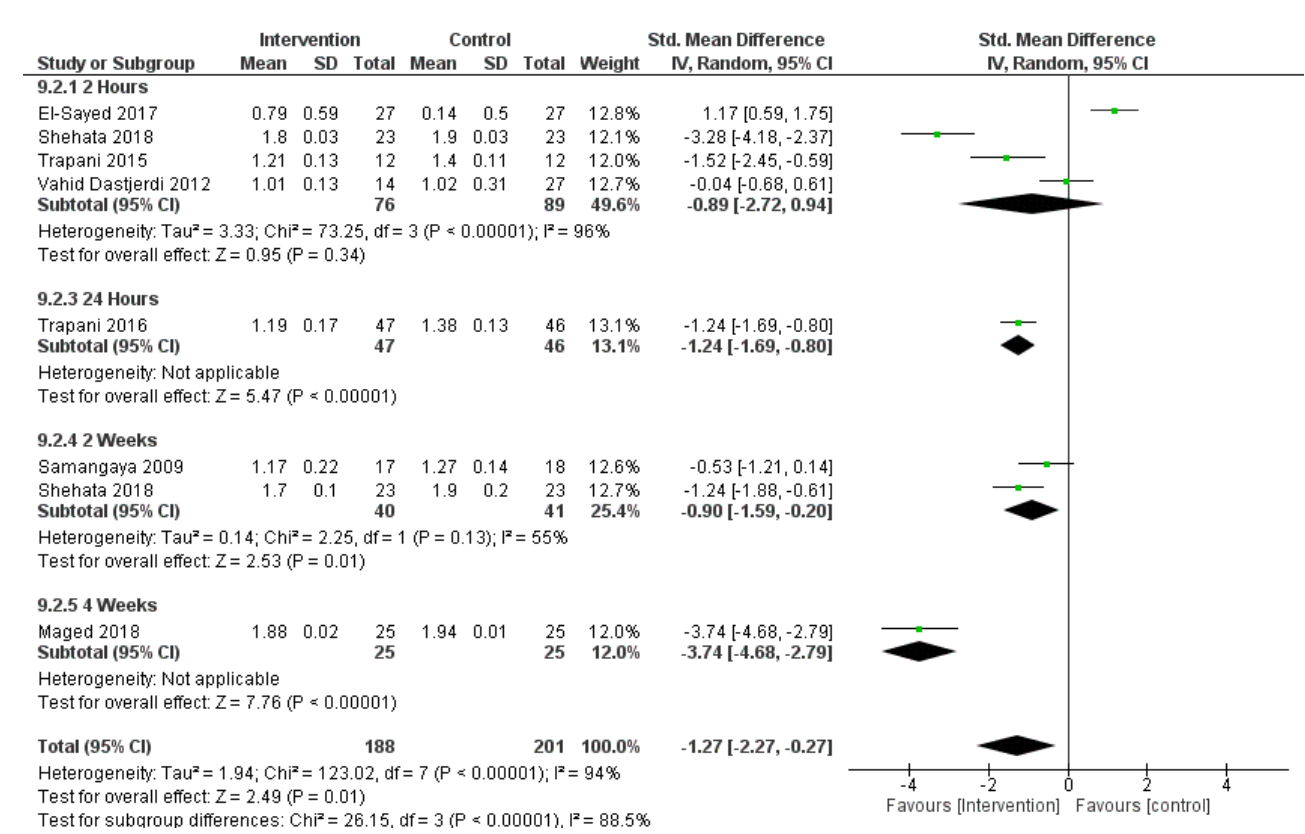

Figure 2. The mean difference of UA PI between sildenafil and the control group based on the assessment time (2 hours, 24 hours, 2 weeks, and 4 weeks after sildenafil use) 


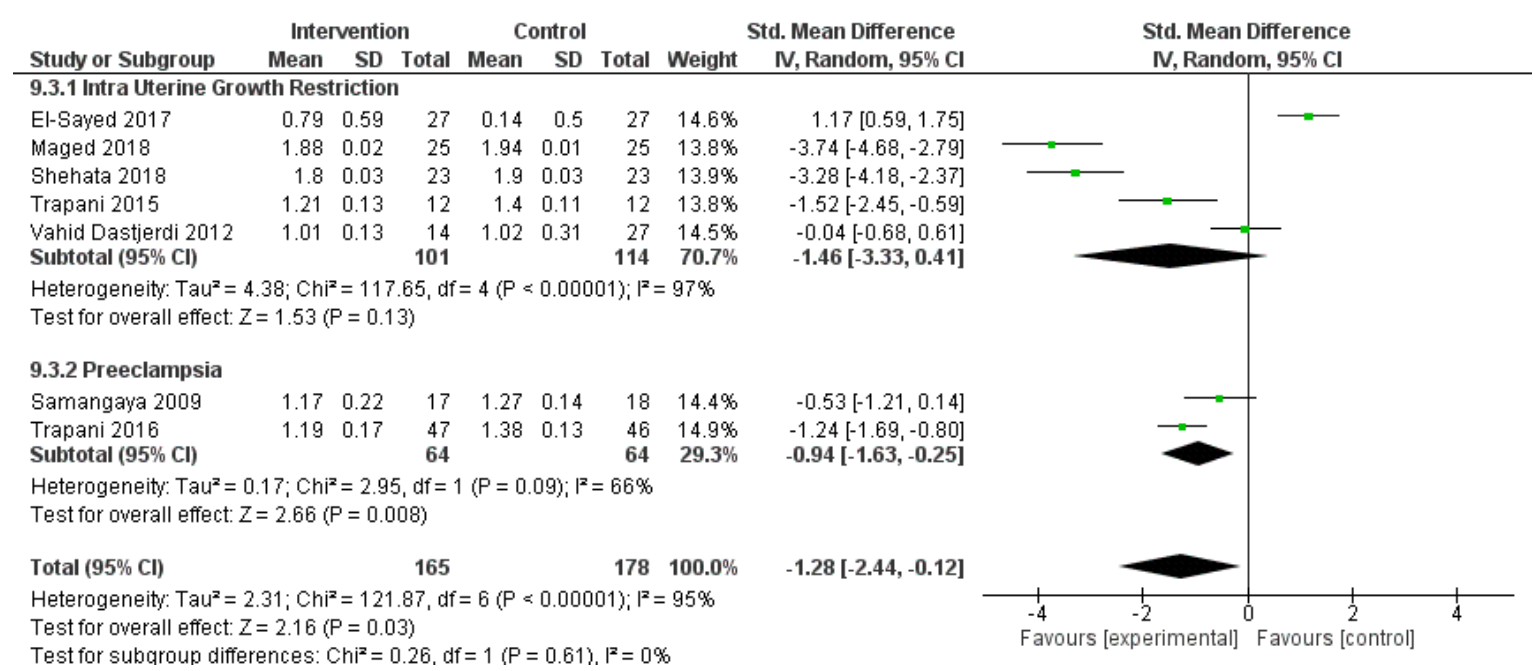

Figure 3. The mean difference of UA PI between sildenafil and the control group in the two subgroups of IUGR and preeclampsia

\section{Middle Cerebral Artery Systolic/Diastolic}

Sildenafil had no effect on the MCA S/D ratio. The effect was significant only at the dosage of 20-60 $\mathrm{mg} / \mathrm{d}(\mathrm{SMD}=1.06 ; 95 \% \mathrm{CI}, 0.49-1.63 ; P<0.001)$ and in high quality studies (SMD $=1.06 ; 95 \% \mathrm{CI}, 0.49$ $1.63 ; P<0.001)$ (Table 2).

The heterogeneity values for different types of studies are as follows: 95\% $(P<0.001)$ for studies conducted on UA PI, 97\% $(P<0.001)$ for studies conducted on the UA S/D ratio, 96\% $(P<0.001)$ for studies conducted on MCA PI, and $77 \%(P=0.04)$ for studies conducted on the MCA S/D ratio. A subgroup analysis decreased the heterogeneity to $55 \%$ in studies conducted on UA PI (Table 3 ).

\section{Adverse Events}

The meta-analysis conducted on maternal adverse events revealed a 3.57-times increase $(95 \%$ CI, 1.14 11.18; $P=0.03$ ) in the occurrence of headache in the intervention group compared to the control group. The other maternal adverse events presented no statistically significant differences between the two groups. Furthermore, no significant heterogeneity existed among the studies conducted on maternal adverse events (Table 4).

Table 3. Heterogeneity assessment using the Chi-square test in different sub-groups of the study (based on I2 statistics)

\begin{tabular}{|c|c|c|c|c|c|c|c|c|}
\hline \multirow{3}{*}{ Heterogeneity } & \multicolumn{4}{|c|}{ Umbilical Artery } & \multicolumn{4}{|c|}{ Middle Cerebral Artery } \\
\hline & \multicolumn{2}{|c|}{ PI } & \multicolumn{2}{|c|}{ S/D ratio } & \multicolumn{2}{|c|}{ PI } & \multicolumn{2}{|c|}{ S/D ratio } \\
\hline & $\mathbf{I}^{2}$ & P-value & $\mathbf{I}^{2}$ & P-value & $\mathbf{I}^{2}$ & P-value & $\mathbf{I}^{2}$ & P-value \\
\hline \multicolumn{9}{|c|}{ Dose of the Sildenafil } \\
\hline $50 \mathrm{mg}$ Single dose & 85 & 0.01 & - & - & 0 & 0.49 & - & - \\
\hline 20 to $60 \mathrm{mg}$ daily & 98 & $<0.001$ & 99 & $<0.001$ & 99 & $<0.001$ & - & - \\
\hline$>$ than $60 \mathrm{mg}$ daily & 66 & 0.09 & - & - & - & - & - & - \\
\hline \multicolumn{9}{|c|}{ Time of outcome assessment } \\
\hline 2 hours & 96 & $<0.001$ & 92 & $<0.001$ & 97 & $<0.001$ & 77 & 0.04 \\
\hline 24 hours & - & - & - & - & - & - & - & - \\
\hline 2 weeks & 55 & 0.13 & - & - & - & - & - & - \\
\hline 4 weeks & - & - & - & - & - & - & - & - \\
\hline \multicolumn{9}{|c|}{ Study population } \\
\hline IUGR & 97 & $<0.001$ & 97 & $<0.001$ & 97 & $<0.001$ & 77 & 0.04 \\
\hline Preeclampsia & 66 & 0.09 & - & - & - & - & - & - \\
\hline \multicolumn{9}{|c|}{ Quality of the studies } \\
\hline High & 94 & $<0.001$ & - & - & 83 & 0.002 & - & - \\
\hline Moderate & - & - & - & - & - & - & - & - \\
\hline Low & 98 & $<0.001$ & 96 & $<0.001$ & - & - & - & - \\
\hline Total effect & 95 & $<0.001$ & 97 & $<0.001$ & 96 & $<0.001$ & 77 & 0.04 \\
\hline
\end{tabular}

Abbreviations: PI, pulsatility index; S/D, systolic/diastolic; IUGR, intrauterine growth restriction. 
Table 4. The effects of sildenafil on the reported maternal adverse events (based on the odds ratio and random effect)

\begin{tabular}{|cccccc}
\hline Adverse events & Odds ratio & Total effects & & \multicolumn{2}{c}{ Heterogeneity } \\
\hline Headache & 3.57 & $1.14-11.18$ & 0.03 & 57 & P-value \\
\hline $\begin{array}{c}\text { Nausea or } \\
\text { Vomiting }\end{array}$ & 1.29 & $0.42-3.93$ & 0.66 & 46 & 0.06 \\
\hline $\begin{array}{c}\text { Flushing } \\
\text { Epigastric pain }\end{array}$ & 4.26 & $0.45-40.58$ & 0.21 & 0 & 0.14 \\
\hline $\begin{array}{c}\text { Diarrhea } \\
\text { Neurological } \\
\text { disturbances }\end{array}$ & 0.73 & $0.33-1.62$ & 0.44 & 0 & 0.83 \\
$\begin{array}{c}\text { Visual } \\
\text { disturbances }\end{array}$ & 0.94 & $0.23-3.82$ & 0.93 & 4 & 0.56 \\
\hline $\begin{array}{c}\text { Dizziness } \\
\text { Back pain }\end{array}$ & 0.91 & $0.22-1.69$ & 0.34 & 0 & 0.35 \\
\hline $\begin{array}{c}\text { Placental } \\
\text { abruption }\end{array}$ & 0.75 & $0.16-3.59$ & 0.72 & 0 & 0.88 \\
$\begin{array}{c}\text { Post-Partum } \\
\text { Hemorrhage }\end{array}$ & 1.10 & $0.18-24.87$ & 0.56 & - & 0.48 \\
$\begin{array}{c}\text { HELLP } \\
\text { syndrome }\end{array}$ & 0.51 & $0.37-12.16$ & 0.40 & 0 & 0.68 \\
\hline Eclampsia & 0.32 & $0.05-13$ & 0.83 & 0 & 0.94 \\
\hline
\end{tabular}

\section{Discussion}

This systematic review summarized the fetal Doppler indices following sildenafil use in the pregnancy in the existence of RCTs. The outcomes are reported separately for UA and MCA indices in IUGR and preeclampsia studies.

The evaluation of UA PI revealed that, in general, UA PI is reduced significantly in pregnant women who used sildenafil compared to the control group.

In this meta-analysis, a sub-analysis was conducted to determine "the effective dosage of sildenafil in UA PI," "the best time for evaluating UA PI after sildenafil use," "the effect size comparison of UA PI on individuals suffering from IUGR and preeclampsia," and "the quality of studies."

Regarding the effective dosage, the obtained data revealed that a significant difference in UA PI is observed only when the dose is increased to more than $60 \mathrm{mg} / \mathrm{d}$. Meanwhile, a significant difference in UA PI was observed by Trapani and Dastjerdi after a single dosage of $50 \mathrm{mg}(\mathbf{1 0}, \mathbf{5})$. As no pharmacokinetic studies on sildenafil during pregnancy are available, it is not possible to discuss the proper dose that should be used in future studies.

Regarding the best time for evaluating the UA PI after sildenafil use, an assessment made two hours after using sildenafil showed no meaningful effects on UA PI. In other words, when the sample size and power of the study were increased, assessments made two hours after the administration of sildenafil did not alter the significance of the effect. However, sildenafil was effective when other assessment times, such as 24 hours, two weeks, or four weeks, were investigated. However, the presence of few existing studies in each group made the results unreliable. Assessments made two hours after using sildenafil have been deemed by most of the studies as the standard time for viewing the effects in UA PI. Such a standard time has been deemed by Trapani to be one to four hours after use, while Shehata made assessments two hours and two weeks after use, and Sharp made assessments one week after use $(10,9,25)$.

The effect size of UA PI on populations suffering from IUGR and preeclampsia showed that UA PI decreased significantly after sildenafil consumption only in individuals suffering from preeclampsia and was insignificant in those suffering from IUGR. This finding yields various interpretations.

The first interpretation is that five out of seven studies focused on IUGR (Figure 3). It seems that, even though UA PI was significant in each of the RCT studies, the significance of the sildenafil effect on individuals suffering from IUGR faded as the sample size and power of the study increased. Therefore, studies with larger populations are required to draw accurate conclusions about the effect of sildenafil on UA PI. In other words, it is possible that if we had more RCTs (and a larger sample size) on preeclampsia, we would not see a significant effect. This finding somewhat conforms 
to those obtained by Sharp's study, in which sildenafil was found to be ineffective in IUGR. The population in Sharp's study (i.e., 65 individuals in the control group and 70 individuals in the intervention group) was larger than those of other RCT studies (25).

The second interpretation stems from the difference in the heterogeneity of the IUGR and preeclampsia studies. In IUGR RCTs, the heterogeneity of the studies was significant. That is, although the studies were different in terms of their procedure, the preeclampsia studies (two RCTs) were similar in design. Sildenafil might have had a significant effect on UA PI in the IUGR group if they were of the same standard; this also could have decreased the heterogeneity.

The assessment of the UA S/D ratio revealed that this index has not changed significantly as a result of sildenafil use. This might be explained through the insignificant effect of sildenafil on IUGR and preeclampsia, which conforms to Sharp's study (25). No significant differences were observed as a result of changing the dose of sildenafil and study population. However, the UA S/D ratio became significant in high-quality studies assessing the outcome four weeks after use.

An evaluation of MCA PI showed that sildenafil did not affect this index as it relates to the embryo. Regarding this index, the effect was significant in medium quality studies at a two-hour follow-up, two-week follow-up, and for IUGR subgroups (Table 2). Sildenafil had no significant effect on the MCA S/D ratio. Furthermore, in general, sildenafil did not affect the MCA S/D ratio. Its effect was significant only for $20-60 \mathrm{mg} / \mathrm{d}$ dosage subgroups in high-quality studies (Table 2).

The meta-analysis conducted on maternal adverse events revealed that sildenafil significantly increased the occurrence of headaches in the intervention group by as much as 3.57 times (95\% CI, $1.14-11.18 ; P=0.03)$ compared to the control group. No statistically significant differences were found for other maternal adverse events between the two groups. The obtained results are of higher credibility, considering that no significant heterogeneity existed in the studies on maternal adverse effects. Conforming to these results, in a systematic review on the effect of sildenafil during pregnancy, Dunn showed that headaches are the most prevalent adverse effect in mothers (prevalence rate of $46 \%)(18)$.

\section{Conclusion}

Before any decision can be made on the effect of sildenafil on fetal Doppler indices and, ultimately, on preeclampsia and IUGR, more high-quality RCTs with adequate study populations are required. On the other hand, more credible results on the effectiveness of sildenafil in preeclampsia and IUGR might be obtained via information registration systems (26).

\section{Acknowledgments}

The authors thank all those who helped them writing this article.

\section{Ethical considerations}

Ethical issues (Including plagiarism, informed consent, misconduct, data fabrication and/or falsification, double publication and/or submission, redundancy, etc.) have been completely observed by the authors.

\section{Funding and support}

This research resulted from an independent research without receiving any financial support.

\section{Conflict of Interest}

Authors declared no conflict of interest.

\section{References}

1. Dashe JS, Bloom SL, Spong CY, Hoffman BL. Williams Obstetrics: McGraw Hill Professional; 2018.

2. Kenny LC, Baker PN, Kendall DA, Randall MD. Differential mechanisms of endotheliumdependent vasodilator responses in human myometrial small arteries in normal pregnancy and pre-eclampsia. Clin Sci. 2002;103(1):67-73. [DOI:10.1042/cs1030067]

3. Zoma W, Baker R, Friedman A, Clark K. Sildenafil citrate (Viagra) increases uterine blood flow and potentiates estrogen-induced vasodilation. J Soc Gynecol Invest. 2001;8.

4. Wareing M, Myers JE, O'Hara M, Baker PN. Sildenafil citrate (Viagra) enhances vasodilatation in fetal growth restriction. J Clin Endocrinol Metab. 2005;90(5):2550-5. [DOI:10.1210/jc.2004-1831]

5. Dastjerdi MV, Hosseini S, Bayani L. Sildenafil citrate and uteroplacental perfusion in fetal growth restriction. J Isfahan Univ Med Sci. 2012;17(7):632.

6. El-Sayed MA, Saleh SA-A, Maher MA, Khidre AM. Utero-placental perfusion doppler indices in growth restricted fetuses: effect of sildenafil citrate. J Maternal Fetal Neonat Med. 2018;31(8):1045-50. [DOI:10.1080/14767058.2017.1306509]

7. Maged M, Wageh A, Shams M, Elmetwally A. Use of sildenafil citrate in cases of intrauterine 
growth restriction (IUGR); a prospective trial. Taiwan J Obstet Gynecol. 2018;57(4):483-6. [DOI:10.1016/j.tjog.2018.06.002]

8. Samangaya RA, Mires G, Shennan A, et al. A randomised, double-blinded, placebo-controlled study of the phosphodiesterase type 5 inhibitor sildenafil for the treatment of preeclampsia. Hypertension Pregnancy. 2009;28(4):369-82. [DOI: $10.3109 / 10641950802601278]$

9. Shehata NA, Ali HA, Fahim AS, Katta MA, Hussein GK. Addition of sildenafil citrate for treatment of severe intrauterine growth restriction: a double blind randomized placebo controlled trial. J Maternal Fetal Neonat Med. 2018;(33)10:1 7. [DOI:10.1080/14767058.2018.1523892]

10. Trapani A, Gonçalves LF, Trapani TF, Vieira S, Pires M, Pires MMdS. Perinatal and hemodynamic evaluation of sildenafil citrate for preeclampsia treatment. Obstet Gynecol. 2016;128(2):253-9. [DOI:10.1097/AOG.0000000000001518]

11. Trapani Jr A, Gonçalves L, Trapani T, Franco M, Galluzzo R, Pires M. Comparison between transdermal nitroglycerin and sildenafil citrate in intrauterine growth restriction: effects on uterine, umbilical and fetal middle cerebral artery pulsatility indices. Ultrasound Obstet Gynecol. 2016;48(1):61-5. [DOI:10.1002/uog.15673]

12. Gillis EE, Mooney JN, Garrett MR, Granger JP, Sasser JM. Sildenafil treatment ameliorates the maternal syndrome of preeclampsia and rescues fetal growth in the dahl salt-sensitive rat. Hypertension. 2016;67(3):647-53. [DOI:10.1161/HYPERTENSIONAHA.115.06071]

13. Cheraghi Z, Esfahani BO, Mohammadian Z, Nooreldinc RS. Prevalence of preeclampsia and eclampsia in Iran. Arch Iran Med. 2016;19(1):64.

14. Li R, Tsigas EZ, Callaghan WM. Health and economic burden of preeclampsia: no time for complacency. Am J Obstet Gynecol. 2017;217(3):235-6.

[DOI:10.1016/j.ajog.2017.06.011]

15. Kharaghani R, Geranmaye M, Janani L, et al. Preeclampsia and depression: a case-control study in Tehran. Arch Gynecol Obstet. 2012;286(1):249-53. [DOI:10.1007/s00404-0122260-3]

16. Ganzevoort W, Alfirevic Z, von Dadelszen P, et al. STRIDER: Sildenafil therapy in dismal prognosis early-onset intrauterine growth restriction-a protocol for a systematic review with individual participant data and aggregate data meta-analysis and trial sequential analysis. Systematic Rev. 2014;3(1):23. [DOI:10.1186/2046-4053-3-23]

17. Villanueva-Garcia D, Mota-Rojas D, HernandezGonzalez R, et al. A systematic review of experimental and clinical studies of sildenafil citrate for intrauterine growth restriction and preterm labour. J Obstet Gynecol. 2007;27(3):255-9. [DOI:10.1080/01443610701194978]

18. Dunn L, Greer R, Flenady V, Kumar S. Sildenafil in pregnancy: a systematic review of maternal tolerance and obstetric and perinatal outcomes. Fetal Diag Ther. 2017;41(2):81-8. [DOI:10.1159/000453062]

19. Morris R, Say R, Robson S, Kleijnen J, Khan K. Systematic review and meta-analysis of middle cerebral artery Doppler to predict perinatal wellbeing. Europ J Obstet Gynecol Reproduct Biol. 2012;165(2):141-55. [DOI:10.1016/j.ejogrb.2012.07.027]

20. Morris R, Malin G, Robson S, Kleijnen J, Zamora J, Khan K. Fetal umbilical artery doppler to predict compromise of fetal/neonatal wellbeing in a highrisk population: systematic review and bivariate meta-analysis. Ultrasound Obstet Gynecol. 2011;37(2):135-42. [DOI:10.1002/uog.7767]

21. Cohen J. A power primer. Psychol Bull. 1992;112(1):155. 2909.112.1.155]

22. Higgins JP, Thompson SG. Quantifying heterogeneity in a meta-analysis. Stat Med. 2002;21(11):1539-58. [DOI:10.1002/sim.1186]

23. Begg CB, Mazumdar M. Operating characteristics of a rank correlation test for publication bias. Biometrics. 1994:1088-101. [DOI:10.2307/2533446]

24. Egger M, Smith GD, Schneider M, Minder C. Bias in meta-analysis detected by a simple, graphical test. BMJ. 1997;315(7109):629-34. [DOI:10.1136/bmj.315.7109.629]

25. Sharp A, Cornforth C, Jackson R, et al. Maternal sildenafil for severe fetal growth restriction (STRIDER): a multicentre, randomised, placebocontrolled, double-blind trial. Lancet Child Adolescent Health. 2018;2(2):93-102. [DOI:10.1016/S2352-4642(17)30173-6]

26. Kharaghani R, Shariati M, Keramat A, Yunesian M, Moghisi A. 9: Adaptation of pregnancy risk assessment monitoring system (PRAMS) and provide a model on IT. BMJ Open. 2017;7(Suppl 1).

\section{How to Cite This Article:}

Damghanian M, Farnam F, Kharaghani R. The Effects of Sildenafil on Fetal Doppler Indices: A Systematic Review and Meta-Analysis. J Adv Med Biomed Res. 2020; 28 (131) :307-315 